\title{
COHOMOLOGY OF FIBER-BUNCHED TWISTED COCYCLES OVER HYPERBOLIC SYSTEMS
}

\author{
LUCAS BACKES
}

\begin{abstract}
A twisted cocycle taking values on a Lie Group $G$ is a cocycle that, in each step, is twisted by an automorphism of $G$. In the case when $G=$ $G L(d, \mathbb{R})$, we prove that if two Hölder continuous twisted cocycles satisfying the so called fiber-bunching condition have the same periodic data then they are cohomologous.
\end{abstract}

\section{INTRODUCTION}

Given a homeomorphism $f: M \rightarrow M$ acting on compact metric space $(M, d)$ and an automorhism $\alpha \in \operatorname{Aut}(G)$ of a topological group $G$, we say that the map $A_{\alpha}: \mathbb{Z} \times M \rightarrow G$ is an $\alpha$-twisted cocycle over $f$ if

$$
A_{\alpha}^{m+n}(x)=A_{\alpha}^{n}\left(f^{m}(x)\right) \alpha^{n}\left(A_{\alpha}^{m}(x)\right)
$$

for all $x \in M$ and $m, n \in \mathbb{Z}$.

Two $\alpha$-twisted cocycles $A_{\alpha}$ and $B_{\alpha}$ over $f$ are said to be $\alpha$-cohomologous whenever there exists a transfer map map $P: M \rightarrow G$ satisfying

$$
A_{\alpha}^{n}(x)=P\left(f^{n}(x)\right) B_{\alpha}^{n}(x) \alpha^{n}(P(x))^{-1}
$$

for every $x \in M$ and $n \in \mathbb{Z}$. Observe that in the case when $\alpha=$ Id the notions of $\alpha$-twisted cocycle and $\alpha$-cohomology coincide with the "standard" notions of cocycles and cohomology in Dynamical Systems [KN11].

Cohomology of twisted cocycles appears naturally in many problems in Dynamics. For instance, any map $A: M \rightarrow G$ naturally generates an $\alpha$-twisted cocycle $A_{\alpha}$ over $f$ (see Section 2.2). In this case, we can consider the twisted skew-product $F_{A, \alpha}: M \times G \rightarrow M \times G$ given by $F_{A, \alpha}(x, g)=(f(x), A(x) \alpha(g))$. Now, the problem of determining whether two twisted skew-products $F_{A, \alpha}$ and $F_{B, \alpha}$ are conjugated reduces to the problem of studying whether $A_{\alpha}$ and $B_{\alpha}$ are $\alpha$-cohomologous. In fact, the map $U(x, g)=(x, P(x) g)$ conjugates $F_{A, \alpha}$ and $F_{B, \alpha}$ precisely when $P$ is a transfer map for $A_{\alpha}$ and $B_{\alpha}$. This observation applied to the case when $G=G L(d, \mathbb{R})$ is what motivates much of this note. Other applications also appear in the study of regularity of the transfer map for non-abelian cocycles over Anosov actions [NT98], in applications to the differentiable rigidity of Anosov diffeomorphisms [dlL87] and the study of local rigidity of higher rank abelian partially hyperbolic actions [DK10]. For more applications we refer to Section 4.6 of [KN11] and to [Kon95].

In the present paper we are interested in describing necessary and sufficient conditions under which two $\alpha$-twisted cocycles $A_{\alpha}$ and $B_{\alpha}$ are $\alpha$-cohomologous

2010 Mathematics Subject Classification. Primary: 37H05, 37A20; Secondary: 37D20.

Key words and phrases. Twisted cocycles, Cohomology, Hyperbolic Systems, Periodic points. 
whenever $f$ is a hyperbolic map. In the case when $\alpha=\operatorname{Id}$ and $G$ is an abelian group admiting a bi-invariant metric, a first criterion was given by Livšic in his seminal papers [Liv71] and [Liv72]. More precisely, he proved that $A_{\text {Id }}$ and $B_{\text {Id }}$ are Id-cohomologous if and only if

$$
A_{\mathrm{Id}}^{n}(p)=B_{\mathrm{Id}}^{n}(p) \text { for every } p \in \operatorname{Fix}\left(f^{n}\right) .
$$

Because of its many applications, still in the case when $\alpha=\mathrm{Id}$, this criterion was extended by many authors to many different settings usually eliminating the assumptions that $G$ is abelian and admits a bi-invariant metric. See for instance [AKL18, Bac15, BK16, Kal11, Par99, Sad15, Sch99].

The case when $\alpha$ is not the identity, on the other hand, despite of its many applications, has received much less attention. To the best of the author's knowledge, the best result in this setting is a theorem by Walkden [Wal00] where he got an analogous result to the original Livšic's theorem under the assumptions that $G$ is a connected Lie group admitting a bi-invariant metric ${ }^{1}$ and the automorphism $\alpha$ satisfies some "growth" conditions. The objective of this paper is to extend the results of [Wal00] to the case when $G=G L(d, \mathbb{R})$.

1.1. Main results. The main result of this work is the following one (see Section 2 for precise definitions):

Theorem 1.1. Let $f: M \rightarrow M$ be a Lipschitz continuous transitive hyperbolic homeomorphism on a compact metric space $(M, d), A, B: M \rightarrow G L(d, \mathbb{R})$ two $\nu$ Hölder continuous maps and $\alpha \in A u t(G L(d, \mathbb{R}))$ be an automorphism of $G L(d, \mathbb{R})$. Suppose that the twisted cocycles $A_{\alpha}$ and $B_{\alpha}$ are fiber-bunched. Moreover, suppose that they satisfy the periodic orbit condition

$$
A_{\alpha}^{n}(p)=B_{\alpha}^{n}(p), \quad \forall n \in \mathbb{Z}, \forall p \in F i x\left(f^{n}\right) .
$$

Then, there exists a $\nu$-Hölder continuous map $P: M \rightarrow G L(d, \mathbb{R})$ such that

$$
A_{\alpha}^{n}(x)=P\left(f^{n}(x)\right) B_{\alpha}^{n}(x) \alpha^{n}(P(x))^{-1}, \quad \forall x \in M, \forall n \in \mathbb{Z} .
$$

This result consists of a generalization of the main results of [Bac15] and [Sad15] to the case of twisted cocycles. In fact, the main result of those works can be obtained as corollaries of the previous one by taking $\alpha=\mathrm{Id}$. Moreover, this result also generalizes the main result of [Wal00] in the case when $G=G L(d, \mathbb{R})$. Indeed, it was observed in [Wal00, Remark 3.4] that in such case, instead asking for the group to admit a bi-invariant metric (recall that $G L(d, \mathbb{R})$ does not admit such a metric), one can assume some bounded distortion condition in the twisted cocycles. Roughly speaking, this condition asks for each of the terms in the left-hand side of (5) to be uniformly bounded. In particular, such condition is much more restrictive than our fiber-bunching assumption.

One can easily see that the $\alpha$-cohomology relation is an equivalence one over the space of $\alpha$-twisted cocycles. In particular, as a simple consequence of the previous result one can get a complete characterization of the cohomology classes in the twisted scenario in terms of the periodic data:

\footnotetext{
${ }^{1}$ In the case when $G=G L(d, \mathbb{R})$ the existence of the bi-invariant metric can be replaced by a bounded distortion condition. See comments after Theorem 1.1
} 
Corollary 1.2. Let $f, A, B$ and $\alpha$ be as in Theorem 1.1 and, moreover, suppose that $A$ or $B$ satisfies (5), (6) and (7) with $7 \rho+2 \theta<\nu \lambda$. Then, there exists a $\nu$-Hölder continuous map $Q: M \rightarrow G L(d, \mathbb{R})$ such that

$$
A_{\alpha}^{n}(p)=Q(p) B_{\alpha}^{n}(p) \alpha^{n}(Q(p))^{-1}
$$

for every $n \in \mathbb{Z}$ and $p \in F i x\left(f^{n}\right)$ if and only if there exists a $\nu$-Hölder continuous map $P: M \rightarrow G L(d, \mathbb{R})$ such that

$$
A_{\alpha}^{n}(x)=P\left(f^{n}(x)\right) B_{\alpha}^{n}(x) \alpha^{n}(P(x))^{-1}, \quad \forall x \in M, \forall n \in \mathbb{Z} .
$$

Proof. One implication is trivial. Let us deduce the other one. Assume that $B$ satisfies (5), (6) and (7) with $7 \rho+2 \theta<\nu \lambda$. The case when $A$ satisfies it is similar. Let us consider

$$
\tilde{B}_{\alpha}^{n}(x)=Q\left(f^{n}(x)\right) B_{\alpha}^{n}(x) \alpha^{n}(Q(x))^{-1} .
$$

We start observing that $\left(\tilde{B}_{\alpha}^{n}\right)_{n \in \mathbb{Z}}$ is an $\alpha$-twisted cocycle over $f$. Indeed,

$$
\begin{aligned}
\tilde{B}_{\alpha}^{n+m}(x) & =Q\left(f^{n+m}(x)\right) B_{\alpha}^{n+m}(x) \alpha^{m+n}(Q(x))^{-1} \\
& =Q\left(f^{n+m}(x)\right) B_{\alpha}^{n}\left(f^{m}(x)\right) \alpha^{n}\left(B_{\alpha}^{m}(x)\right) \alpha^{m+n}(Q(x))^{-1} \\
& =Q\left(f^{n+m}(x)\right) B_{\alpha}^{n}\left(f^{m}(x)\right) \alpha^{n}\left(Q\left(f^{m}(x)\right)^{-1} Q\left(f^{m}(x)\right)\right) \alpha^{n}\left(B_{\alpha}^{m}(x)\right) \alpha^{m+n}(Q(x))^{-1} \\
& =Q\left(f^{n+m}(x)\right) B_{\alpha}^{n}\left(f^{m}(x)\right) \alpha^{n}\left(Q\left(f^{m}(x)\right)^{-1} \alpha^{n}\left(Q\left(f^{m}(x)\right) B_{\alpha}^{m}(x) \alpha^{m}(Q(x))^{-1}\right)\right. \\
& =\tilde{B}_{\alpha}^{n}\left(f^{m}(x)\right) \alpha^{n}\left(\tilde{B}_{\alpha}^{m}(x)\right) .
\end{aligned}
$$

Moreover, our hypothesis on $B$ ensures that $\tilde{B}$ is fiber-bunched in the sense of Section 2.5. Thus, since $A_{\alpha}^{n}(p)=\tilde{B}_{\alpha}^{n}(p)$ for every $p \in \operatorname{Fix}\left(f^{n}\right)$ the result follows by applying our main result to these two cocycles.

Observe that the previous proof gives us no apparent "meaningful" relation between the maps $P$ and $Q$ given in the statement of Corollary 1.2.

In order to proof our main result we follow the approaches of [Bac15], which in turn was inspired by [Par99, Sch99], and [Sad15, Wal00]. The main idea consists in constructing invariant holonomies, which is a family of linear maps with good properties (see Proposition 3.1), and then, using this family, to explicitly construct the transfer map on a dense set under the additional assumption that $f$ admits a fixed point. The next step consists in showing that, restricted to this dense set, the transfer map is $\nu$-Hölder continuous and then extending it to the whole space. Finally, we explain how to eliminate the hypothesis of existence of a fixed point for $f$. The main difference from this proof and the one in [Bac15] is that the estimates here are much more involved due to the presence of twisting. The overall strategy is the same. In particular, the last step of the proof is the same, mutatis mutandis, as in the untwisted case and so we only indicate how to proceed.

Throughout the paper we are going to use the letter $C$ as a generic notation for a positive constant that may change from line to line. Whenever necessary, we will explicitly mention the parameters on which $C$ depends.

\section{Preliminaries}

Let $(M, d)$ be a compact metric space, $f: M \rightarrow M$ a homeomorphism, $G$ a Lie group and $A: M \rightarrow G$ a $\nu$-Hölder continuous map. 
2.1. Hyperbolic homeomorphisms. Given any $x \in M$ and $\varepsilon>0$, define the local stable and unstable sets of $x$ with respect to $f$ by

$$
\begin{aligned}
& W_{\varepsilon}^{s}(x):=\left\{y \in M: d\left(f^{n}(x), f^{n}(y)\right) \leq \varepsilon, \forall n \geq 0\right\}, \\
& W_{\varepsilon}^{u}(x):=\left\{y \in M: d\left(f^{n}(x), f^{n}(y)\right) \leq \varepsilon, \forall n \leq 0\right\},
\end{aligned}
$$

respectively. Following [AV10], we introduce the following

Definition 2.1. A homeomorphism $f: M \rightarrow M$ is said to be hyperbolic with local product structure (or just hyperbolic for short) whenever there exist constants $C, \varepsilon, \lambda, \tau>0$ such that the following conditions are satisfied:

$\circ d\left(f^{n}\left(y_{1}\right), f^{n}\left(y_{2}\right)\right) \leq C e^{-\lambda n} d\left(y_{1}, y_{2}\right), \forall x \in M, \forall y_{1}, y_{2} \in W_{\varepsilon}^{s}(x), \forall n \geq 0 ;$

$\circ d\left(f^{-n}\left(y_{1}\right), f^{-n}\left(y_{2}\right)\right) \leq C e^{-\lambda n} d\left(y_{1}, y_{2}\right), \forall x \in M, \forall y_{1}, y_{2} \in W_{\varepsilon}^{u}(x), \forall n \geq 0$;

- If $d(x, y) \leq \tau$, then $W_{\varepsilon}^{s}(x)$ and $W_{\varepsilon}^{u}(y)$ intersect in a unique point which is denoted by $[x, y]$ and depends continuously on $x$ and $y$.

For such homeomorphisms, one can define the stable and unstable sets by

$$
W^{s}(x):=\bigcup_{n \geq 0} f^{-n}\left(W_{\varepsilon}^{s}\left(f^{n}(x)\right)\right) \text { and } W^{u}(x):=\bigcup_{n \geq 0} f^{n}\left(W_{\varepsilon}^{u}\left(f^{-n}(x)\right)\right),
$$

respectively.

Notice that subshifts of finite type and basic sets of Axiom A diffeomorphisms are particular examples of hyperbolic homeomorphisms with local product structure (see for instance [Mañ87, Chapter IV,§ 9] for details).

2.2. Twisted Cocycles. Let Aut $(G)$ denote the group of automorphisms of $G$ and $\alpha \in \operatorname{Aut}(G)$. A map $A_{\alpha}: \mathbb{Z} \times M \rightarrow G$ is said to be an $\alpha$-twisted cocycle over $f$ if

$$
A_{\alpha}^{m+n}(x)=A_{\alpha}^{n}\left(f^{m}(x)\right) \alpha^{n}\left(A_{\alpha}^{m}(x)\right)
$$

for all $x \in M$ and $m, n \in \mathbb{Z}$. To any map $A: M \rightarrow G$ we may associate an $\alpha$-twisted cocycle over $f$ by

$$
A_{\alpha}^{n}(x)= \begin{cases}A\left(f^{n-1}(x)\right) \alpha\left(A\left(f^{n-2}(x)\right)\right) \ldots \alpha^{n-2}(A(f(x))) \alpha^{n-1}(A(x)) & \text { if } n>0 \\ \operatorname{Id} & \text { if } n=0 \\ \alpha^{n}\left(A_{\alpha}^{-n}\left(f^{n}(x)\right)^{-1}\right) & \text { if } n<0\end{cases}
$$

for all $x \in M$. In this case we say that $A$ generates the $\alpha$-twisted cocycle $A_{\alpha}$ over $f$. Reciprocally, every $\alpha$-twisted cocycle $A_{\alpha}$ is generated by $A=A_{\alpha}^{1}$. In what follows, for sake of simplicity, we write just $A_{\alpha}$ instead of $A_{\alpha}^{1}$.

2.3. Cohomology of $\alpha$-twisted cocycles. Given a $\nu$-Hölder continuous map $B$ : $M \rightarrow G$, we say that the $\alpha$-twisted cocycles $A_{\alpha}$ and $B_{\alpha}$ generated by $A$ and $B$ over $f$, respectively, are $\alpha$-cohomologous if there exists a $\nu$-Hölder continuous map $P: M \rightarrow G$ such that

$$
A_{\alpha}(x)=P(f(x)) B_{\alpha}(x) \alpha(P(x))^{-1}
$$

for every $x \in M$. It is easy to verify that this equation is equivalent to

$$
A_{\alpha}^{n}(x)=P\left(f^{n}(x)\right) B_{\alpha}^{n}(x) \alpha^{n}(P(x))^{-1}
$$

for every $x \in M$ and $n \in \mathbb{Z}$. As already observed in the introduction, whenever $\alpha=$ Id we recover the usual notions of cocycles and cohomology [Bac15, Sad15]. 
2.4. Linear $\alpha$-twisted cocycles. From now on we restrict ourselves to the case when $G=G L(d, \mathbb{R})$. In particular, by $A: M \rightarrow G L(d, \mathbb{R})$ being $\nu$-Hölder continuous we mean that there exists a constant $C>0$ such that

$$
\|A(x)-A(y)\| \leq C d(x, y)^{\nu}
$$

for all $x, y \in M$ where $\|A\|$ denotes the operator norm of a matrix $A$, that is, $\|A\|=\sup \{\|A v\| /\|v\| ;\|v\| \neq 0\}$.

Observe that examples of automorphisms of $G L(d, \mathbb{R})$ are $\alpha_{L}: G L(d, \mathbb{R}) \rightarrow$ $G L(d, \mathbb{R})$ and $\alpha_{i}: G L(d, \mathbb{R}) \rightarrow G L(d, \mathbb{R})$ given by

$$
\alpha_{L}(A)=L A L^{-1} \text { and } \alpha_{i}(A)=\left(A^{T}\right)^{-1}
$$

where $L \in G L(d, \mathbb{R})$ is a fixed matrix and $A^{T}$ denotes the transpose of $A$. For more on $\operatorname{Aut}(G L(d, \mathbb{R}))$ we refer to $[\operatorname{Mcd} 78]$.

2.5. Fiber-bunched $\alpha$-twisted cocycles. We say that the $\alpha$-twisted cocycle $A_{\alpha}$ generated by $A$ over $f$ is fiber-bunched if there are constants $C>0$ and $\rho, \theta>0$ with $5 \rho+2 \theta<\nu \lambda$, where $\nu$ and $\lambda$ are as in (4) and Definition 2.1, respectively, such that for every $n \in \mathbb{Z}$,

i)

$$
\left\|\alpha^{-n}\left(A_{\alpha}^{n}(x)\right)\right\|\left\|\alpha^{-n}\left(A_{\alpha}^{n}(x)^{-1}\right)\right\|<C e^{\theta|n|}
$$

ii)

for every $x \in M$;

$$
\left\|\alpha^{n}\left(T_{1}\right)-\alpha^{n}\left(T_{2}\right)\right\| \leq C e^{\rho|n|}\left\|T_{1}-T_{2}\right\|
$$
iii)

for every $T_{1}, T_{2} \in G L(d, \mathbb{R})$;

$$
\left\|\alpha^{n}(T)\right\| \leq C e^{\rho|n|}\|T\|
$$

for every $T \in G L(d, \mathbb{R})$.

Once again, it is easy to see that by taking $\alpha=$ Id we recover the "standard" notion of fiber-bunched cocycles used for instance in [AV10, Bac15, BGV03, Sad15].

Observe that if $A$ and $\alpha$ are sufficiently close to the identity then the fiberbunching condition is automatically satisfied. Other examples of $\alpha$-twisted cocycles with $\alpha \neq$ Id satisfying the fiber-bunching condition are given, for instance, by taking $\alpha=\alpha_{L}$ as in the previous subsection with $L$ close enough to Id and assuming the cocycle $(A, f)$ is fiber-bunched in the standard sense of [BGV03, Via08]. It is also worth noticing that this fiber-bunching notion is related to the partial hyperbolicity of the map $F_{A, \alpha}: M \times G \rightarrow M \times G$ given by $F_{A, \alpha}(x, g)=(f(x), A(x) \alpha(g))$. Indeed, condition (5) says that the rates of expansion and contraction given by $F_{A, \alpha}$ along the $G$-direction are "dominated" by the rates of expansion and contraction along the $M$-direction.

\section{INVARIANT HOLONOMIES}

In this section we introduce the notion of invariant holonomies for twisted cocycles. This is done by generalizing the notion introduced by [BGV03, Via08] in the untwisted case. As in the untwisted scenario, these objects are fundamental in our proof. 
Proposition 3.1. Let $f: M \rightarrow M$ be a hyperbolic homeomorphism on a compact metric space $(M, d), A: M \rightarrow G L(d, \mathbb{R})$ be a $\nu$-Hölder map and $\alpha \in \operatorname{Aut}(G)$. Suppose that the twisted cocycle $A_{\alpha}$ generated by $A$ and $\alpha$ over $f$ is fiber-bunched. Then there exists a constant $C=C(A, \alpha, f)>0$ such that, for any $x \in M$ and any $y, z \in W^{s}(x)$ the limit

$$
H_{y z}^{s, A, \alpha}:=\lim _{n \rightarrow+\infty} \alpha^{-n}\left(A_{\alpha}^{n}(z)^{-1} A_{\alpha}^{n}(y)\right)
$$

exists and

$$
\left\|H_{y z}^{s, A, \alpha}-I d\right\| \leq C d(y, z)^{\nu},
$$

whenever $y, z \in W_{\varepsilon}^{s}(x)$, where the constant $\varepsilon>0$ associated to $f$ is given by Definition 2.1.

On the other hand, if $y, z \in W^{u}(x)$, we can analogously define

$$
H_{y z}^{u, A, \alpha}:=\lim _{n \rightarrow+\infty} \alpha^{n}\left(A_{\alpha}^{-n}(z)^{-1} A_{\alpha}^{-n}(y)\right),
$$

and the very same Hölder estimates holds for these maps when $y, z \in W_{\varepsilon}^{u}(x)$.

Finally, for every $x \in M$ and $* \in\{s, u\}$, it holds

$$
H_{y z}^{*, A, \alpha}=H_{x z}^{*, A, \alpha} H_{y x}^{*, A, \alpha},
$$

and

$$
H_{f^{m}(y) f^{m}(z)}^{*, A, \alpha}=A_{\alpha}^{m}(z) \alpha^{m}\left(H_{y z}^{*, A, \alpha}\right) A_{\alpha}^{m}(y)^{-1},
$$

for every $y, z \in W^{*}(x)$ and $m \in \mathbb{Z}$.

Definition 3.2. The maps $H^{s, A, \alpha}$ and $H^{u, A, \alpha}$ given by Proposition 3.1 are called stable and unstable holonomies, respectively.

It is worth noticing that the main ideas beyond this concept, even though not under this name, were somehow present in [Wal00] (see also [Par99, Sch99] for the case $\alpha=I d)$. On the other hand, the construction of this holonomies in that setting is greatly simplified due to the existence of a bi-invariant metric. Similarly, the proof in the case $\alpha=\mathrm{Id}$ is also much simpler when compared to ours due to the lack of twisting (see for instance Proposition 2.5 of [Via08]).

We will prove only the assertions about $H_{y z}^{s, A, \alpha}$ since the ones about $H_{y z}^{u, A, \alpha}$ are similar. We start with the following proposition:

Proposition 3.3. Let $\delta>0$ be so that $5 \rho+2 \theta+\delta<\lambda \nu$. Then, there exists $C=C(A, \alpha, f, \delta)>0$ such that

$$
\left\|\alpha^{-n}\left(A_{\alpha}^{n}(y)\right)\right\| \cdot\left\|\alpha^{-n}\left(A_{\alpha}^{n}(x)^{-1}\right)\right\| \leq C e^{(4 \rho+2 \theta+\delta) n}
$$

for all $y \in W_{\varepsilon}^{s}(x), x \in M$ and $n \geq 0$.

In order to prove this proposition we need a couple of auxiliary results.

Lemma 3.4. Fix $x \in M$. There exists a family of norms $\left(\|\cdot\|_{k}\right)_{k \in \mathbb{N}}$ such that

$$
\frac{\max \left\{\left\|\alpha^{-k}\left(A\left(f^{k-1}(x)\right)\right) v\right\|_{k} ;\|v\|_{k-1}=1\right\}}{\min \left\{\left\|\alpha^{-k}\left(A\left(f^{k-1}(x)\right)\right) w\right\|_{k} ;\|w\|_{k-1}=1\right\}} \leq e^{2 \theta+\delta} .
$$

Moreover, there exists $C>0$ depending only on $A, \alpha, f$ and $\delta$ so that

$$
\|\cdot\| \leq\|\cdot\|_{k} \leq C e^{2 \rho k}\|\cdot\| \text { for every } k \in \mathbb{N} .
$$


Proof. Fix $u_{0} \in \mathbb{R}^{d}$ with $\left\|u_{0}\right\|=1$ and for any $k \in \mathbb{Z}$ set

$$
u_{k}=\frac{\alpha^{-k}\left(A\left(f^{k-1}(x)\right)\right) u_{k-1}}{\left\|\alpha^{-k}\left(A\left(f^{k-1}(x)\right)\right) u_{k-1}\right\|} .
$$

Now, given $v \in \mathbb{R}^{d}$ define

$$
\|v\|_{k}^{2}=\sum_{m \in \mathbb{Z}} \frac{\left\|\alpha^{-m-k}\left(A_{\alpha}^{m}\left(f^{k}(x)\right)\right) v\right\|^{2}}{\left\|\alpha^{-m-k}\left(A_{\alpha}^{m}\left(f^{k}(x)\right)\right) u_{k}\right\|^{2} \cdot e^{(2 \theta+\delta)|m|}} .
$$

We start observing that from (7)

$$
\left\|\alpha^{-m-k}\left(A_{\alpha}^{m}\left(f^{k}(x)\right)\right) \frac{v}{\|v\|}\right\|\left\|\alpha^{-m-k}\left(A_{\alpha}^{m}\left(f^{k}(x)\right)\right) u_{k}\right\|^{-1}
$$

is smaller than or equal to

$$
C e^{2 \rho k}\left\|\alpha^{-m}\left(A_{\alpha}^{m}\left(f^{k}(x)\right)\right) \frac{v}{\|v\|}\right\|\left\|\alpha^{-m}\left(A_{\alpha}^{m}\left(f^{k}(x)\right)\right) u_{k}\right\|^{-1} .
$$

Thus, using our hypothesis (5) and the fact that $\|T\|^{-1} \leq\left\|T^{-1}\right\|$ for any $T \in$ $G L(d, \mathbb{R})$ we get that the last quantity is smaller than or equal to $C^{2} e^{2 \rho k} e^{\theta|m|}$. In particular,

$$
\left\|\alpha^{-m-k}\left(A_{\alpha}^{m}\left(f^{k}(x)\right)\right) v\right\|\left\|\alpha^{-m-k}\left(A_{\alpha}^{m}\left(f^{k}(x)\right)\right) u_{k}\right\|^{-1} \leq C^{2} e^{2 \rho k} e^{\theta|m|}\|v\|
$$

for every $m \in \mathbb{Z}$ and thus

$$
\|v\|_{k}^{2} \leq \sum_{m \in \mathbb{Z}} \frac{\left(C^{2} e^{2 \rho k} e^{\theta|m|}\|v\|\right)^{2}}{e^{(2 \theta+\delta)|m|}} \leq \tilde{C} e^{4 \rho k}\|v\|^{2}
$$

where $\tilde{C}=\sum_{m \in \mathbb{Z}} C^{4} e^{-\delta|m|}<\infty$. Consequently, the series (10) converges and $\|\cdot\|_{k}$ is well defined. Moreover

$$
\|v\|_{k} \leq C e^{2 \rho k}\|v\|
$$

for any $v \in \mathbb{R}^{d}$ and some $C>0$ independent of $k$ and $x$. Furthermore, recalling that $\alpha^{-k}(\mathrm{Id})=\mathrm{Id}$ and $\left\|u_{k}\right\|=1$, looking at the term of (10) when $m=0$ it follows that $\|v\| \leq\|v\|_{k}$ for every $v \in \mathbb{R}^{d}$ which combined with the previous observations completes the proof of (9). In order to prove the other claim, we observe that

$$
\begin{aligned}
\left\|\alpha^{-k}\left(A\left(f^{k-1}(x)\right)\right) v\right\|_{k}^{2} & =\sum_{m \in \mathbb{Z}} \frac{\left\|\alpha^{-m-k}\left(A_{\alpha}^{m}\left(f^{k}(x)\right)\right) \alpha^{-k}\left(A\left(f^{k-1}(x)\right)\right) v\right\|^{2}}{\left\|\alpha^{-m-k}\left(A_{\alpha}^{m}\left(f^{k}(x)\right)\right) u_{k}\right\|^{2} \cdot e^{(2 \theta+\delta)|m|}} \\
& =\sum_{m \in \mathbb{Z}} \frac{\left\|\alpha^{-m-k}\left(A_{\alpha}^{m}\left(f^{k}(x)\right)\right) \alpha^{-k}\left(A\left(f^{k-1}(x)\right)\right) v\right\|^{2}}{\left\|\alpha^{-m-k}\left(A_{\alpha}^{m}\left(f^{k}(x)\right)\right)\left(\frac{\alpha^{-k}\left(A\left(f^{k-1}(x)\right)\right) u_{k-1}}{\left\|\alpha^{-k}\left(A\left(f^{k-1}(x)\right)\right) u_{k-1}\right\|}\right)\right\|^{2} \cdot e^{(2 \theta+\delta)|m|}} \\
& =\sum_{m \in \mathbb{Z}} \frac{\left\|\alpha^{-m-k}\left(A_{\alpha}^{m+1}\left(f^{k-1}(x)\right)\right) v\right\|^{2}\left\|\alpha^{-k}\left(A\left(f^{k-1}(x)\right)\right) u_{k-1}\right\|^{2}}{\left\|\alpha^{-m-k}\left(A_{\alpha}^{m+1}\left(f^{k-1}(x)\right)\right) u_{k-1}\right\|^{2} \cdot e^{(2 \theta+\delta)|m|}} \\
& =\left\|\alpha^{-k}\left(A\left(f^{k-1}(x)\right)\right) u_{k-1}\right\|^{2} \cdot S(v)
\end{aligned}
$$

where

$$
S(v):=\sum_{m \in \mathbb{Z}} \frac{\left\|\alpha^{-(m+1)-(k-1)}\left(A_{\alpha}^{m+1}\left(f^{k-1}(x)\right)\right) v\right\|^{2}}{\left\|\alpha^{-(m+1)-(k-1)}\left(A_{\alpha}^{m+1}\left(f^{k-1}(x)\right)\right) u_{k-1}\right\|^{2} \cdot e^{(2 \theta+\delta)|m|}} .
$$


Now, since $|m+1| \geq|m|-1$, we get that $S(v) \leq e^{2 \theta+\delta}\|v\|_{k-1}^{2}$. Similarly, since $|m+1| \leq|m|+1$, we get that $S(v) \geq e^{-(2 \theta+\delta)}\|v\|_{k-1}^{2}$. Combining these facts with the previous observations it follows that

$$
\begin{aligned}
e^{-\left(\theta+\frac{\delta}{2}\right)}\left\|\alpha^{-k}\left(A\left(f^{k-1}(x)\right)\right) u_{k-1}\right\|\|v\|_{k-1} & \leq\left\|\alpha^{-k}\left(A\left(f^{k-1}(x)\right)\right) v\right\|_{k} \\
& \leq e^{\theta+\frac{\delta}{2}}\left\|\alpha^{-k}\left(A\left(f^{k-1}(x)\right)\right) u_{k-1}\right\|\|v\|_{k-1}
\end{aligned}
$$

for any $v \in \mathbb{R}^{d}$. Thus, taking $v, w \in \mathbb{R}^{d}$ so that $\|v\|_{k-1}=\|w\|_{k-1}=1$ it follows that

$$
\begin{aligned}
e^{-(2 \theta+\delta)}\left\|\alpha^{-k}\left(A\left(f^{k-1}(x)\right)\right) v\right\|_{k} & \leq\left\|\alpha^{-k}\left(A\left(f^{k-1}(x)\right)\right) w\right\|_{k} \\
& \leq e^{2 \theta+\delta}\left\|\alpha^{-k}\left(A\left(f^{k-1}(x)\right)\right) v\right\|_{k} .
\end{aligned}
$$

Consequently,

$$
\frac{\max \left\{\left\|\alpha^{-k}\left(A\left(f^{k-1}(x)\right)\right) v\right\|_{k} ;\|v\|_{k-1}=1\right\}}{\min \left\{\left\|\alpha^{-k}\left(A\left(f^{k-1}(x)\right)\right) w\right\|_{k} ;\|w\|_{k-1}=1\right\}} \leq e^{2 \theta+\delta}
$$

as claimed.

Thus, defining the $k$-norm of an operator $T \in G L(d, \mathbb{R})$ with respect to the family of norms $\left(\|\cdot\|_{k}\right)_{k \in \mathbb{N}}$ by

$$
\|T\|_{k}=\sup _{v \neq 0} \frac{\|T v\|_{k}}{\|v\|_{k-1}}
$$

it follows easily from the previous lemma that

Corollary 3.5. For any $k \in \mathbb{N}$,

$$
\left\|\alpha^{-k}\left(A\left(f^{k-1}(x)\right)\right)^{-1}\right\|_{k}\left\|\alpha^{-k}\left(A\left(f^{k-1}(x)\right)\right)\right\|_{k} \leq e^{2 \theta+\delta} .
$$

Proof of Proposition 3.3. Let $\left(\|\cdot\|_{k}\right)_{k \in \mathbb{Z}}$ be the family of norms given by Lemma 3.4. Recalling (6), (7) and (9), we start observing that

$$
\begin{aligned}
\frac{\left\|\alpha^{-k}\left(A\left(f^{k-1}(y)\right)\right)\right\|_{k}}{\left\|\alpha^{-k}\left(A\left(f^{k-1}(x)\right)\right)\right\|_{k}} & \leq 1+\frac{\left|\left\|\alpha^{-k}\left(A\left(f^{k-1}(y)\right)\right)\right\|_{k}-\left\|\alpha^{-k}\left(A\left(f^{k-1}(x)\right)\right)\right\|_{k}\right|}{\left\|\alpha^{-k}\left(A\left(f^{k-1}(x)\right)\right)\right\|_{k}} \\
& \leq 1+\frac{\left\|\alpha^{-k}\left(A\left(f^{k-1}(y)\right)\right)-\alpha^{-k}\left(A\left(f^{k-1}(x)\right)\right)\right\|_{k}}{\left\|\alpha^{-k}\left(A\left(f^{k-1}(x)\right)\right)\right\|_{k}} \\
& \leq 1+\frac{C e^{2 \rho k}\left\|\alpha^{-k}\left(A\left(f^{k-1}(y)\right)\right)-\alpha^{-k}\left(A\left(f^{k-1}(x)\right)\right)\right\|}{\left\|\alpha^{-k}\left(A\left(f^{k-1}(x)\right)\right)\right\|} \\
& \leq 1+\frac{C e^{4 \rho k}\left\|A\left(f^{k-1}(y)\right)-A\left(f^{k-1}(x)\right)\right\|}{\left\|A\left(f^{k-1}(x)\right)\right\|} .
\end{aligned}
$$

Thus, since $A$ is $\nu$-Hölder and $M$ is compact and recalling Definition 2.1 it follows that

$$
\frac{\left\|\alpha^{-k}\left(A\left(f^{k-1}(y)\right)\right)\right\|_{k}}{\left\|\alpha^{-k}\left(A\left(f^{k-1}(x)\right)\right)\right\|_{k}} \leq 1+C e^{(4 \rho-\lambda \nu) k} d(x, y)^{\nu} .
$$

Now, Corollary 3.5 gives us that for any $j \in \mathbb{N}$,

$$
\left\|\alpha^{-j}\left(A\left(f^{j-1}(x)\right)\right)^{-1}\right\|_{j} \leq \frac{e^{2 \theta+\delta}}{\left\|\alpha^{-j}\left(A\left(f^{j-1}(x)\right)\right)\right\|_{j}} .
$$


Combining these two observations with the fact that

$$
\begin{aligned}
\left\|\alpha^{-k}\left(A_{\alpha}^{k}(x)\right)^{-1}\right\|_{k} & =\left\|\alpha^{-1}(A(x))^{-1} \alpha^{-2}(A(f(x)))^{-1} \ldots \alpha^{-k}\left(A\left(f^{k-1}(x)\right)\right)^{-1}\right\|_{k} \\
& \leq\left\|\alpha^{-1}(A(x))^{-1}\right\|_{1}\left\|\alpha^{-2}(A(f(x)))^{-1}\right\|_{2} \ldots\left\|\alpha^{-k}\left(A\left(f^{k-1}(x)\right)\right)^{-1}\right\|_{k}
\end{aligned}
$$

and similarly

$$
\left\|\alpha^{-k}\left(A_{\alpha}^{k}(y)\right)\right\|_{k} \leq\left\|\alpha^{-k}\left(A\left(f^{k-1}(y)\right)\right)\right\|_{k} \ldots\left\|\alpha^{-2}(A(f(x)))\right\|_{2}\left\|\alpha^{-1}(A(x))\right\|_{1}
$$

it follows that

$$
\begin{aligned}
\left\|\alpha^{-k}\left(A_{\alpha}^{k}(x)\right)^{-1}\right\|_{k}\left\|\alpha^{-k}\left(A_{\alpha}^{k}(y)\right)\right\|_{k} & \leq \frac{\left\|\alpha^{-1}(A(y))\right\|_{1}}{\left\|\alpha^{-1}(A(x))\right\|_{1}} e^{2 \theta+\delta} \cdots \frac{\left\|\alpha^{-k}\left(A\left(f^{k-1}(y)\right)\right)\right\|_{k}}{\left\|\alpha^{-k}\left(A\left(f^{k-1}(x)\right)\right)\right\|_{k}} e^{2 \theta+\delta} \\
& \leq e^{(2 \theta+\delta) k} \prod_{j=1}^{k}\left(1+C e^{(4 \rho-\lambda \nu) j} d(x, y)^{\nu}\right) \\
& \leq \tilde{C} e^{(2 \theta+\delta) k}
\end{aligned}
$$

where $\tilde{C}=\prod_{j=1}^{\infty}\left(1+C D e^{(4 \rho-\lambda \nu) j}\right)<\infty$ and $D=\sup _{x, y \in M} d(x, y)^{\nu}$ (recall that $4 \rho-\lambda \nu<0)$. Thus, since by (9) we have that $\|T\| \leq C e^{2 \rho k}\|T\|_{k}$ for every $T \in$ $G L(d, \mathbb{R})$ it follows that

$$
\left\|\alpha^{-k}\left(A_{\alpha}^{k}(x)\right)^{-1}\right\|\left\|\alpha^{-k}\left(A_{\alpha}^{k}(y)\right)\right\| \leq C e^{(4 \rho+2 \theta+\delta) k}
$$

for some constant $C$ independent of $x$ and $y$ as claimed.

We are now ready to prove the main proposition of this section.

Proof of Proposition 3.1. By taking forward iterates we can assume that $y, z \in$ $W_{\frac{\varepsilon}{2}}^{u}(x)$. In particular, $z \in W_{\varepsilon}^{s}(y)$. We are going to show that the sequence $\left(\alpha^{-n}\left(A_{\alpha}^{n}(z)^{-1} A_{\alpha}^{n}(y)\right)\right)_{n}$ is a Cauchy sequence. In order to do it we start observing that for every $n \in \mathbb{N}$,

$$
\left\|\alpha^{-(n+1)}\left(A_{\alpha}^{n+1}(z)^{-1} A_{\alpha}^{n+1}(y)\right)-\alpha^{-n}\left(A_{\alpha}^{n}(z)^{-1} A_{\alpha}^{n}(y)\right)\right\|
$$

is equal to

$\left\|\alpha^{-n}\left(A_{\alpha}^{n}(z)^{-1}\right) \alpha^{-(n+1)}\left(A\left(f^{n}(z)\right)^{-1} A\left(f^{n}(y)\right)\right) \alpha^{-n}\left(A_{\alpha}^{n}(y)\right)-\alpha^{-n}\left(A_{\alpha}^{n}(z)^{-1}\right) \alpha^{-n}\left(A_{\alpha}^{n}(y)\right)\right\|$

which is smaller than or equal to

$$
\left\|\alpha^{-n}\left(A_{\alpha}^{n}(z)^{-1}\right)\right\|\left\|\alpha^{-n}\left(A_{\alpha}^{n}(y)\right)\right\|\left\|\alpha^{-(n+1)}\left(A\left(f^{n}(z)\right)^{-1} A\left(f^{n}(y)\right)\right)-\mathrm{Id}\right\| .
$$

From Proposition 3.3 it follows that the previous quantity is smaller than or equal to

$$
C e^{(4 \rho+2 \theta+\delta) n}\left\|\alpha^{-(n+1)}\left(A\left(f^{n}(z)\right)^{-1} A\left(f^{n}(y)\right)\right)-\mathrm{Id}\right\| .
$$

Thus, since

$$
\begin{aligned}
\left\|\alpha^{-(n+1)}\left(A\left(f^{n}(z)\right)^{-1} A\left(f^{n}(y)\right)\right)-\mathrm{Id}\right\| & =\left\|\alpha^{-(n+1)}\left(A\left(f^{n}(z)\right)^{-1} A\left(f^{n}(y)\right)\right)-\alpha^{-(n+1)}(\mathrm{Id})\right\| \\
& \leq C e^{\rho(n+1)}\left\|A\left(f^{n}(z)\right)^{-1} A\left(f^{n}(y)\right)-\mathrm{Id}\right\| \\
& \leq C e^{\rho(n+1)} e^{-\nu \lambda n} d(z, y)^{\nu} \\
& =C e^{\rho} e^{(\rho-\nu \lambda) n} d(z, y)^{\nu}
\end{aligned}
$$


we get that

$$
\begin{aligned}
\left\|\alpha^{-(n+1)}\left(A_{\alpha}^{n+1}(z)^{-1} A_{\alpha}^{n+1}(y)\right)-\alpha^{-n}\left(A_{\alpha}^{n}(z)^{-1} A_{\alpha}^{n}(y)\right)\right\| & \leq C e^{(4 \rho+2 \theta+\delta) n} C e^{\rho} e^{(\rho-\nu \lambda) n} d(z, y)^{\nu} \\
& =C e^{(5 \rho+2 \theta+\delta-\nu \lambda) n} d(z, y)^{\nu} .
\end{aligned}
$$

Therefore, since $5 \rho+2 \theta+\delta-\nu \lambda<0$, we get that the sequence $\left(\alpha^{-n}\left(A_{\alpha}^{n}(z)^{-1} A_{\alpha}^{n}(y)\right)\right)_{n}$ is indeed a Cauchy sequence. Consequently

$$
H_{y z}^{s, A, \alpha}=\lim _{n \rightarrow+\infty} \alpha^{-n}\left(A_{\alpha}^{n}(z)^{-1} A_{\alpha}^{n}(y)\right)
$$

exists and moreover

$$
\left\|H_{y z}^{s, A, \alpha}-\mathrm{Id}\right\| \leq C d(y, z)^{\nu}
$$

whenever $y, z \in W_{\varepsilon}^{s}(x)$ as claimed.

To prove the last claim we start observing that, on the one hand,

$$
\alpha^{-n}\left(A_{\alpha}^{n}(z)^{-1} A_{\alpha}^{n}(y)\right) \stackrel{n \rightarrow \infty}{\longrightarrow} H_{y z}^{s, A, \alpha} .
$$

On the other hand,

$$
\alpha^{-n}\left(A_{\alpha}^{n}(z)^{-1} A_{\alpha}^{n}(y)\right)
$$

is equal to

$$
\alpha^{-m}\left(A_{\alpha}^{m}(z)^{-1}\right) \alpha^{-m}\left(\alpha^{-(n-m)}\left(A_{\alpha}^{n-m}\left(f^{m}(z)\right)^{-1} A_{\alpha}^{n-m}\left(f^{m}(y)\right)\right)\right) \alpha^{-m}\left(A_{\alpha}^{m}(y)\right)
$$

which converges to

$$
\alpha^{-m}\left(A_{\alpha}^{m}(z)^{-1}\right) \alpha^{-m}\left(H_{f^{m}(y) f^{m}(z)}^{s, A, \alpha}\right) \alpha^{-m}\left(A_{\alpha}^{m}(y)\right)
$$

as $n$ goes to infinity. Combining these observations we conclude that

$$
H_{f^{m}(y) f^{m}(z)}^{s, \alpha}=A_{\alpha}^{m}(z) \alpha^{m}\left(H_{y z}^{s, A, \alpha}\right) A_{\alpha}^{m}(y)^{-1},
$$

as claimed.

Remark 3.6. From the proof of Proposition 3.3 we can easily see that in order to get

$$
\left\|\alpha^{-k}\left(A_{\alpha}^{k}(y)\right)\right\| \cdot\left\|\alpha^{-k}\left(A_{\alpha}^{k}(x)^{-1}\right)\right\| \leq C e^{(4 \rho+2 \theta+\delta) k}
$$

for every $0 \leq k \leq n$ we don't actually need $y \in W_{\varepsilon}^{s}(x)$. In fact, we only need $x$ and $y$ to satisfy $d\left(f^{k}(x), f^{k}(y)\right) \leq C e^{-\gamma k} d(x, y)$ for every $0 \leq k \leq n$ and some $\gamma \in(0, \lambda)$ satisfying $4 \rho+\delta<\nu \gamma$. In this case, the constant $C$ will depend on $A, \alpha, f, \delta$ and $\gamma$. We are going to use this fact in the sequel.

The notions of fiber-bunching and invariant holonomies in the case when $\alpha=\mathrm{Id}$ have been playing an important role in many subareas of Dynamical Systems and arise naturally in various different contexts (for instance, [AV10, Bac15, BBB18, BGV03, Sad15, Via08]). Therefore, Proposition 3.1 is also likely to have many applications and can be seen as interesting in itself.

In order to simplify notation, in what follows, whenever $\alpha$ is fixed and there is no ambiguity, we simply write $H^{*, A}$ instead of $H^{*, A, \alpha}$, for $*=s, u$, to denote the stable and unstable holonomy associated to $A_{\alpha}$. 


\section{Constructing the transfer map}

In this section we are going to build "explicitly" the transfer map. The method we use is similar to that used in [Bac15, BK16] and [Sad15] in the untwisted setting and by [Wal00] in the twisted one: using the invariant holonomies we define the transfer map on a dense set, prove that restricted to it, it is Hölder continuous and then extend it to the closure getting the desired result.

Assume there exists $x \in M$ such that $f(x)=x$. For such a point, we write $W(x):=W^{s}(x) \cap W^{u}(x)$. We start defining $P: W(x) \rightarrow G L(d, \mathbb{R})$ by

$$
P(y)=H_{x y}^{s, A}\left(H_{x y}^{s, B}\right)^{-1}=H_{x y}^{s, A} H_{y x}^{s, B},
$$

where $H^{s, A}$ and $H^{s, B}$ are the holonomy maps given by Proposition 3.1 associated to the twisted cocycles $A_{\alpha}$ and $B_{\alpha}$, respectively.

Note that $P$ satisfies

$$
A_{\alpha}^{n}(y)=P\left(f^{n}(y)\right) B_{\alpha}^{n}(y) \alpha^{n}\left(P(y)^{-1}\right)
$$

for every $y \in W(x)$ and every $n \in \mathbb{N}$. Indeed, using that $f(x)=x$, Proposition 3.1 and the hypothesis on periodic points (2),

$$
\begin{aligned}
P\left(f^{n}(y)\right) & =H_{x f^{n}(y)}^{s, A} H_{f^{n}(y) x}^{s, B}=H_{f^{n}(x) f^{n}(y)}^{s, A} H_{f^{n}(y) f^{n}(x)}^{s, B} \\
& =A_{\alpha}^{n}(y) \alpha^{n}\left(H_{x y}^{s, A}\right) A_{\alpha}^{n}(x)^{-1} B_{\alpha}^{n}(x) \alpha^{n}\left(H_{y x}^{s, B}\right) B_{\alpha}^{n}(y)^{-1} \\
& =A_{\alpha}^{n}(y) \alpha^{n}\left(H_{x y}^{s, A} H_{y x}^{s, B}\right) B_{\alpha}^{n}(y)^{-1} \\
& =A_{\alpha}^{n}(y) \alpha^{n}(P(y)) B_{\alpha}^{n}(y)^{-1}
\end{aligned}
$$

and thus

$$
A_{\alpha}^{n}(y)=P\left(f^{n}(y)\right) B_{\alpha}^{n}(y) \alpha^{n}\left(P(y)^{-1}\right)
$$

as claimed.

We will now show that $P$ is $\nu$-Hölder continuous. This will allow us to extend $P$ to $\overline{W(x)}=M$ and thus to get the desired transfer map. The main ingredient in the proof is the next lemma which says that $P$ can be interchangeably defined using stable or unstable holonomies. Its proof is similar to the one of [Bac15, Lemma 3] and we only present the full details of it because of its main role in our proof and also because the presence of twist makes some estimates a little more involved than in the untwisted case.

Lemma 4.1. For every $y \in W(x)$,

$$
P(y)=H_{x y}^{s, A} H_{y x}^{s, B}=H_{x y}^{u, A} H_{y x}^{u, B} .
$$

The following classical result (see for instance [KH95, Corollary 6.4.17]) will be used in the proof:

Lemma 4.2 (Anosov Closing Lemma). Given $\gamma \in(0, \lambda)$ there exist $C>0$ and $\varepsilon_{0}>0$ such that if $z \in M$ satisfy $d\left(f^{n}(z), z\right)<\varepsilon_{0}$ then there exists a periodic point $p \in M$ such that $f^{n}(p)=p$ and

$$
d\left(f^{j}(z), f^{j}(p)\right) \leq C e^{-\gamma \min \{j, n-j\}} d\left(f^{n}(z), z\right)
$$

for every $j=0,1, \ldots, n$.

Proof of Lemma 4.1. Let $\delta>0$ be so that $5 \rho+2 \theta+\delta<\lambda \nu$ and $\gamma \in(0, \lambda)$ such that $5 \rho+2 \theta+\delta<\gamma \nu$. Let $C>0$ and $\varepsilon_{0}>0$ be given by the Anosov Closing Lemma associated to $\gamma$. 
Fix an arbitrary point $y \in W(x)$. We begin by noticing that, as $y \in W(x)$, there exist $C>0$ and $n_{0} \in \mathbb{N}$ such that for all $n \geq n_{0}$ we have

$$
d\left(f^{-n}(y), f^{n}(y)\right) \leq C e^{-\lambda\left(n-n_{0}\right)} .
$$

In fact, this follows from the fact that, as $y \in W(x)=W^{s}(x) \cap W^{u}(x)$, there exists $n_{0} \in \mathbb{N}$ such that $f^{n_{0}}(y) \in W_{\varepsilon}^{s}(x)$ and $f^{-n_{0}}(y) \in W_{\varepsilon}^{u}(x)$ and the exponential convergence towards $x$ in $W_{\varepsilon}^{s}(x)$ and $W_{\varepsilon}^{u}(x)$.

Let $n_{1} \geq n_{0}$ be such that, for all $n \geq n_{1}, d\left(f^{n}(y), f^{-n}(y)\right)<\varepsilon_{0}$. Thus, by the Anosov Closing Lemma, for every $n \geq n_{1}$ there exists a periodic point $p_{n} \in M$ with $f^{2 n}\left(p_{n}\right)=p_{n}$ such that

$$
d\left(f^{j}\left(f^{-n}\left(p_{n}\right)\right), f^{j}\left(f^{-n}(y)\right) \leq C e^{-\gamma \min \{j, 2 n-j\}} d\left(f^{-n}(y), f^{n}(y)\right)\right.
$$

for every $j=0,1, \ldots, 2 n$. Using the periodic orbit condition (2) and noticing that $f^{2 n}\left(f^{-n}\left(p_{n}\right)\right)=f^{-n}\left(p_{n}\right)$, we get

$$
A_{\alpha}^{2 n}\left(f^{-n}\left(p_{n}\right)\right)=B_{\alpha}^{2 n}\left(f^{-n}\left(p_{n}\right)\right),
$$

which can be rewritten as

$$
A_{\alpha}^{n}\left(p_{n}\right) \alpha^{n}\left(A_{\alpha}^{n}\left(f^{-n}\left(p_{n}\right)\right)\right)=B_{\alpha}^{n}\left(p_{n}\right) \alpha^{n}\left(B_{\alpha}^{n}\left(f^{-n}\left(p_{n}\right)\right)\right),
$$

or, equivalently, as

$$
\alpha^{n}\left(A_{\alpha}^{n}\left(f^{-n}\left(p_{n}\right)\right) B_{\alpha}^{n}\left(f^{-n}\left(p_{n}\right)\right)^{-1}\right)=A_{\alpha}^{n}\left(p_{n}\right)^{-1} B_{\alpha}^{n}\left(p_{n}\right) .
$$

Thus, observing that

$$
A_{\alpha}^{n}\left(f^{-n}\left(p_{n}\right)\right)=\alpha^{n}\left(A_{\alpha}^{-n}\left(p_{n}\right)^{-1}\right)
$$

and similarly

$$
B_{\alpha}^{n}\left(f^{-n}\left(p_{n}\right)\right)^{-1}=\alpha^{n}\left(B_{\alpha}^{-n}\left(p_{n}\right)\right)
$$

we get

$$
\alpha^{n}\left(A_{\alpha}^{-n}\left(p_{n}\right)^{-1} B_{\alpha}^{-n}\left(p_{n}\right)\right)=\alpha^{-n}\left(A_{\alpha}^{n}\left(p_{n}\right)^{-1} B_{\alpha}^{n}\left(p_{n}\right)\right) .
$$

Now we claim that

$$
\left\|\alpha^{-n}\left(A_{\alpha}^{n}(y)^{-1} B_{\alpha}^{n}(y)\right)-\alpha^{-n}\left(A_{\alpha}^{n}\left(p_{n}\right)^{-1} B_{\alpha}^{n}\left(p_{n}\right)\right)\right\| \stackrel{n \rightarrow+\infty}{\longrightarrow} 0
$$

and

$$
\left\|\alpha^{n}\left(A_{\alpha}^{-n}(y)^{-1} B_{\alpha}^{-n}(y)\right)-\alpha^{n}\left(A_{\alpha}^{-n}\left(p_{n}\right)^{-1} B_{\alpha}^{-n}\left(p_{n}\right)\right)\right\| \stackrel{n \rightarrow+\infty}{\longrightarrow} 0 .
$$

Consequently, it follows from (12) and our claim that

$$
\left\|\alpha^{-n}\left(A_{\alpha}^{n}(y)^{-1} B_{\alpha}^{n}(y)\right)-\alpha^{n}\left(A_{\alpha}^{-n}(y)^{-1} B_{\alpha}^{-n}(y)\right)\right\| \stackrel{n \rightarrow+\infty}{\longrightarrow} 0 .
$$

Thus, observing that

$$
\alpha^{-n}\left(A_{\alpha}^{n}(y)^{-1} B_{\alpha}^{n}(y)\right)=\alpha^{-n}\left(A_{\alpha}^{n}(y)^{-1} A_{\alpha}^{n}(x) B_{\alpha}^{n}(x)^{-1} B_{\alpha}^{n}(y)\right) \stackrel{n \rightarrow+\infty}{\longrightarrow} H_{x y}^{s, A} H_{y x}^{s, B}
$$

and similarly

we conclude that

$$
\alpha^{n}\left(A_{\alpha}^{-n}(y)^{-1} B_{\alpha}^{-n}(y)\right) \stackrel{n \rightarrow+\infty}{\longrightarrow} H_{x y}^{u, A} H_{y x}^{u, B}
$$

as we wanted.

$$
P(y)=H_{x y}^{s, A} H_{y x}^{s, B}=H_{x y}^{u, A} H_{y x}^{u, B}
$$

So, in order to complete the proof, it remains to prove our claim. We shall only prove (13) since (14) is completely analogous.

We start observing that

$$
\left\|\alpha^{-n}\left(A_{\alpha}^{n}(y) A_{\alpha}^{n}\left(p_{n}\right)^{-1}\right)-\mathrm{Id}\right\|
$$


is smaller than or equal to

$$
\begin{aligned}
\sum_{j=0}^{n-1} \| \alpha^{-(n-j)}\left(A_{\alpha}^{n-j}\left(f^{j}(y)\right)\right. & \left.A_{\alpha}^{n-j}\left(f^{j}\left(p_{n}\right)\right)^{-1}\right) \\
& -\alpha^{-(n-j)}\left(A_{\alpha}^{n-j-1}\left(f^{j+1}(y)\right) A_{\alpha}^{n-j-1}\left(f^{j+1}\left(p_{n}\right)\right)^{-1}\right) \|
\end{aligned}
$$

which by the cocycle property (1) is equal to

$$
\begin{gathered}
\sum_{j=0}^{n-1} \| \alpha^{-(n-j)}\left(A_{\alpha}^{n-j-1}\left(f^{j+1}(y)\right)\right) \alpha^{-1}\left(A\left(f^{j}(y)\right) A\left(f^{j}\left(p_{n}\right)\right)^{-1}\right) \alpha^{-(n-j)}\left(A_{\alpha}^{n-j-1}\left(f^{j+1}\left(p_{n}\right)\right)^{-1}\right) \\
-\alpha^{-(n-j)}\left(A_{\alpha}^{n-j-1}\left(f^{j+1}(y)\right) A_{\alpha}^{n-j-1}\left(f^{j+1}\left(p_{n}\right)\right)^{-1}\right) \| .
\end{gathered}
$$

By the property of the norm this last quantity is smaller than or equal to

$$
\begin{aligned}
\sum_{j=0}^{n-1}\left\|\alpha^{-(n-j)}\left(A_{\alpha}^{n-j-1}\left(f^{j+1}(y)\right)\right)\right\|\left\|\alpha^{-(n-j)}\left(A_{\alpha}^{n-j-1}\left(f^{j+1}\left(p_{n}\right)\right)^{-1}\right)\right\| \\
\cdot\left\|\alpha^{-1}\left(A\left(f^{j}(y)\right) A\left(f^{j}\left(p_{n}\right)\right)^{-1}\right)-\mathrm{Id}\right\|
\end{aligned}
$$

which in its turn is smaller than or equal to

$$
\begin{array}{r}
\sum_{j=0}^{n-1} C^{2} e^{2 \rho}\left\|\alpha^{-(n-j-1)}\left(A_{\alpha}^{n-j-1}\left(f^{j+1}(y)\right)\right)\right\|\left\|\alpha^{-(n-j-1)}\left(A_{\alpha}^{n-j-1}\left(f^{j+1}\left(p_{n}\right)\right)^{-1}\right)\right\| \\
\cdot\left\|A\left(f^{j}(y)\right) A\left(f^{j}\left(p_{n}\right)\right)^{-1}-\mathrm{Id}\right\| .
\end{array}
$$

Now, using Remark 3.6, the fact that $A$ is $\nu$-Hölder continuous and property (11) given by the Anosov Closing Lemma, it follows that the previous quantity is smaller than or equal to

$$
\sum_{j=0}^{n-1} C^{2} e^{2 \rho} C e^{(4 \rho+2 \theta+\delta)(n-j-1)} C e^{-\gamma \nu(n-j-1)} d\left(f^{-n}(y), f^{n}(y)\right)^{\nu} .
$$

Recalling that $d\left(f^{-n}(y), f^{n}(y)\right) \leq e^{-\lambda\left(n-n_{0}\right)}$ for every $n \geq n_{0}$ and $5 \rho+2 \theta+\delta<\gamma \nu$ it follows that

$$
\left\|\alpha^{-n}\left(A_{\alpha}^{n}(y) A_{\alpha}^{n}\left(p_{n}\right)^{-1}\right)-\operatorname{Id}\right\| \leq C e^{-\lambda \nu\left(n-n_{0}\right)}
$$

for every $n \geq n_{0}$ for some constant $C>0$ independent of $n$ and $p_{n}$. Similarly, we can prove that

$$
\left\|\alpha^{-n}\left(B_{\alpha}^{n}\left(p_{n}\right) A_{\alpha}^{n}(y)^{-1}\right)-\mathrm{Id}\right\| \leq C e^{-\lambda \nu\left(n-n_{0}\right)}
$$

for every $n \geq n_{0}$.

Now, using that there exists $N>0$ so that $\left\|\alpha^{-n}\left(A_{\alpha}^{n}(y)^{-1} A_{\alpha}^{n}(x)\right)\right\|<N$ and $\left\|\alpha^{-n}\left(B_{\alpha}^{n}(x)^{-1} B_{\alpha}^{n}(y)\right)\right\|<N$ for every sufficiently large $n$ since these two quantities converge to $H_{x y}^{s, A}$ and $H_{y x}^{s, B}$, respectively; $\left\|\alpha^{-n}\left(A_{\alpha}^{n}(y) A_{\alpha}^{n}\left(p_{n}\right)^{-1}\right)\right\|<N$ for every sufficiently large $n$ by $(15) ; A_{\alpha}^{n}(x)=B_{\alpha}^{n}(x)$ since $A_{\alpha}$ and $B_{\alpha}$ satisfy periodic orbit 
condition (2) and $f(x)=x$; and using (5), (15) and (16), we get

$$
\begin{aligned}
& \left\|\alpha^{-n}\left(A_{\alpha}^{n}(y)^{-1} B_{\alpha}^{n}(y)\right)-\alpha^{-n}\left(A_{\alpha}^{n}\left(p_{n}\right)^{-1} B_{\alpha}^{n}\left(p_{n}\right)\right)\right\| \\
& =\left\|\alpha^{-n}\left(A_{\alpha}^{n}(y)^{-1} A_{\alpha}^{n}(x)\right) \alpha^{-n}\left(B_{\alpha}^{n}(x)^{-1} B_{\alpha}^{n}(y)\right)-\alpha^{-n}\left(A_{\alpha}^{n}\left(p_{n}\right)^{-1} B_{\alpha}^{n}\left(p_{n}\right)\right)\right\| \\
& \leq N^{2}\left\|\operatorname{Id}-\alpha^{-n}\left(A_{\alpha}^{n}(x)^{-1} A_{\alpha}^{n}(y) A_{\alpha}^{n}\left(p_{n}\right)^{-1} B_{\alpha}^{n}\left(p_{n}\right) B_{\alpha}^{n}(y)^{-1} B_{\alpha}^{n}(x)\right)\right\| \\
& \leq N^{2}\left\|\alpha^{-n}\left(A_{\alpha}^{n}(x)^{-1}\right)\right\|\left\|\alpha^{-n}\left(A_{\alpha}^{n}(x)\right)\right\|\left\|\operatorname{Id}-\alpha^{-n}\left(A_{\alpha}^{n}(y) A_{\alpha}^{n}\left(p_{n}\right)^{-1} B_{\alpha}^{n}\left(p_{n}\right) B_{\alpha}^{n}(y)^{-1}\right)\right\| \\
& \leq N^{2} C e^{\theta n}\left(\left\|\alpha^{-n}\left(A_{\alpha}^{n}(y) A_{\alpha}^{n}\left(p_{n}\right)^{-1}\right)\right\|\left\|\alpha^{-n}\left(B_{\alpha}^{n}\left(p_{n}\right) B_{\alpha}^{n}(y)^{-1}\right)-\operatorname{Id}\right\|\right. \\
& \left.+\left\|\alpha^{-n}\left(A_{\alpha}^{n}(y) A_{\alpha}^{n}\left(p_{n}\right)^{-1}\right)-\operatorname{Id}\right\|\right) \\
& \leq N^{2} C e^{\theta n}\left(N C e^{-\lambda \nu\left(n-n_{0}\right)}+C e^{-\lambda \nu\left(n-n_{0}\right)}\right) \\
& \leq \tilde{C} e^{(\theta-\lambda) n}
\end{aligned}
$$

for some $\tilde{C}>0$ independent of $n$ and $p_{n}$ and $n \gg 0$. In particular,

$$
\left\|\alpha^{-n}\left(A_{\alpha}^{n}(y)^{-1} B_{\alpha}^{n}(y)\right)-\alpha^{-n}\left(A_{\alpha}^{n}\left(p_{n}\right)^{-1} B_{\alpha}^{n}\left(p_{n}\right)\right)\right\| \stackrel{n \rightarrow+\infty}{\longrightarrow} 0
$$

proving (13) and thus completing the proof Lemma 4.1.

Lemma 4.3. $P$ is $\nu$-Hölder continuous on $W(x)$.

Proof. The proof of this fact is analogous to the proof of [Bac15, Lemma 4] and so we just summarize the idea beyond it. Full details can be checked in the original work.

From Lemma 4.1 we know that $P$ can be defined using both stable and unstable holonomies. By property (8) we get that restricted to local stable or unstable manifolds, $P$ is Hölder continuous with an uniform Hölder constant. Now, since $f$ has local product structure, points that are $\tau$-close (where $\tau$ is as in Definition 2.1) can be connected via local stable and unstable manifolds. Putting all these facts together we conclude that $P$ is Hölder continuous on balls of radius $\tau$. Finally, using the compactness of $M$ we conclude that $P$ is Hölder continuous in $W(x)$.

Therefore, we can extend $P: W(x) \rightarrow G L(d, \mathbb{R}))$ to the closure of $W(x)$ that is the whole space $M$. By continuity, such extension clearly satisfies the cohomological equation (3) completing the proof of Theorem 1.1 in the case when $f$ exhibits a fixed point.

Now, following the argument given in Section 5 of [Bac15], mutatis mutandis, we eliminate the additional assumption about the existence of a fixed point for $f$ and conclude the proof of Theorem 1.1.

Acknowledgements. Thanks to the referee for the constructive comments on the first version of the paper. The author was partially supported by a CNPq-Brazil PQ fellowship under Grant No. 306484/2018-8.

\section{REFERENCES}

[AKL18] A. Avila, A. Kocsard and X. Liu, Livšic theorem for diffeomorphism cocycles, Geom. Funct. Anal. 28 (2018), 943-964.

[AV10] A. Avila and M. Viana, Extremal Lyapunov exponents: an invariance principle and applications, Invent. Math. 181 (2010), 115-189.

[Bac15] L. Backes, Rigidity of fiber bunched cocycles, Bulletin of the Brazilian Mathematical Society, 46 (2015), 163-179. 
[BBB18] L. Backes, A. Brown and C. Butler, Continuity of Lyapunov exponents for cocycles with invariant holonomies, J. Mod. Dyn. 12 (2018), 223-260.

[BK16] L. Backes and A. Kocsard, Cohomology of dominated diffeomorphism-valued cocycles over hyperbolic systems, Ergodic Theory and Dynamical Systems, 36, (2016), 1703-1722.

[BGV03] C. Bonatti, X. Gómez-Mont and M. Viana, Généricité d'exposants de Lyapunov nonnuls pour des produits déterministes de matrices, Ann. Inst. H. Poincaré Anal. Non Linéaire, 20 (2003), 579-624.

[DK10] D. Damjanović and A. Katok, Local rigidity of partially hyperbolic actions I. KAM method and $\mathbb{Z}^{k}$ actions on the torus, Ann. of Math., 172 (2010), 1805-1858.

[dlL87] R. de la Llave. Invariants for smooth conjugacy of hyperbolic dynamical systems. I. Comm. Math. Phys. 109 (1987), 369-378.

[Kal11] B. Kalinin, Livšic theorem for matrix cocycles. Annals of Mathematics, 173 (2011), 10251042.

[KH95] A. Katok and B. Hasselblatt, Introduction to the modern theory of dynamical systems, Cambridge University Press, London-New York, 1995.

[KN11] A. Katok and V. Niţică, Rigidity in higher rank abelian group actions. Volume I. Introduction and cocycle problem, Cambridge Tracts in Mathematics, vol. 185, Cambridge University Press, Cambridge, 2011.

[Kon95] A. Kononenko, Twisted cocycles and rigidity problems, Electron. Res. Announc. Amer. Math. Soc. 1 (1995), 26-34.

[Liv71] A. Livšic, Homology properties of Y-systems, Math. Zametki 10 (1971), 758-763.

[Liv72] A. Livšic, Cohomology of dynamical systems, Math. USSR Izvestija 6 (1972), 1278-1301.

[Mcd78] R. B. McDonald, Automorphisms of $G L_{n}(R)$, Transactions of the American Mathematical Society 246 (1978), 155-171.

[Mañ87] R. Mañé, Ergodic theory and differentiable dynamics, Ergebnisse der Mathematik und ihrer Grenzgebiete (3), vol. 8, Springer-Verlag, Berlin, 1987, Translated from the Portuguese by Silvio Levy.

[NT98] V. Niţică and A. Török, Regularity of the transfer map for cohomologous cocycles, Ergodic Theory Dynam. Systems 18 (1998), 1187-1209.

[Par99] W. Parry, The Livšic periodic point theorem for non-Abelian cocycles, Ergod. Th. \& Dynam. Sys., 19 (1999), 687-701.

[Sad15] V. Sadovskaya, Cohomology of fiber bunched cocycles over hyperbolic systems, Ergod. Th. \& Dynam. Sys. 35 (2015), 2669-2688.

[Sch99] K. Schmidt, Remarks on Livšic theory for non-Abelian cocycles. Ergod. Th. \& Dynam. Sys. 19 (1999), 703-721.

[Via08] M. Viana, Almost all cocycles over any hyperbolic system have nonvanishing Lyapunov exponents, Annals of Mathematics, 167 (2008), 643-680.

[Wal00] C. Walkden, Solutions to the twisted cocycle equation over hyperbolic systems, Discrete Contin. Dynam. Systems 6 (2000), 935-946.

Departamento de Matemática, Universidade Federal do Rio Grande do Sul, Av. Bento Gonçalves 9500, CeP 91509-900, Porto Alegre, RS, Brazil.

E-mail address: lucas.backes@ufrgs.br 